\title{
Model Reduction of Large-Scale Dynamical Systems
}

A. Antoulas ${ }^{1}$, D. Sorensen ${ }^{2}$, K.A. Gallivan ${ }^{3}$, P. Van Dooren ${ }^{4}$, A. Grama ${ }^{5}$, C. Hoffmann ${ }^{5}$, and A. Sameh ${ }^{5}$

1 Department of Electrical Engineering, Rice University, Houston, TX

2 Department of Computational and Applied Math, Rice University, Houston, TX

3 School of Computational Science, and Information Technology, Florida State University, Tallahassee, FL

4 Department of Mathematical Engineering, Catholic University of Louvain, Louvain-la-Neuve, BELGIUM

5 Department of Computer Sciences, Purdue University, W. Lafayette, IN

\begin{abstract}
Simulation and control are two critical elements of Dynamic Data-Driven Application Systems (DDDAS). Simulation of dynamical systems such as weather phenomena, when augmented with real-time data, can yield precise forecasts. In other applications such as structural control, the presence of real-time data relating to system state can enable robust active control. In each case, there is an ever increasing need for improved accuracy, which leads to models of higher complexity. The basic motivation for system approximation is the need, in many instances, for a simplified model of a dynamical system, which captures the main features of the original complex model. This need arises from limited computational capability, accuracy of measured data, and storage capacity. The simplified model may then be used in place of the original complex model, either for simulation and prediction, or active control. As sensor networks and embedded processors proliferate our environment, technologies for such approximations and real-time control emerge as the next major technical challenge. This paper outlines the state of the art and outstanding challenges in the development of efficient and robust methods for producing reduced order models of large state-space systems.
\end{abstract}

\section{Introduction}

Many physical processes in science and engineering are modeled accurately using finite dimensional dynamical systems. Examples include weather simulation, molecular dynamic simulations (e.g., modeling of bio-molecules and their identification), structural dynamics (e.g., flex models of the international space station, and structural response of high-rise buildings to wind and earthquakes), electronic circuit simulation, semiconductor device manufacturing (e.g., chemical vapor deposition reactors), and simulation and control of micro-electromechanical (MEMS) devices. An important subclass of these processes can be effectively monitored to gather data in support of simulation, diagnosis, prognosis, 
prediction, and control. This class of dynamic data-driven application systems (DDDAS) pose challenging problems ranging from data gathering, assimilation, effective incorporation into simulations, and real-time control.

In the vast majority of applications where control can be affected, the original system is augmented with a second dynamical system called a controller. In general, the controller has the same complexity as the system to be controlled. Since in large-scale applications of interest, one often aims at real-time control; reduced complexity controllers are required. One possible solution to building low-complexity controllers is to design such controllers based on reduced order models of the original dynamical systems. In yet other systems in which realtime prognostics and prediction are required, constraints on compute power, memory, communication bandwidth, and available data might necessitate the use of such reduced-order models. This paper outlines the state-of-the-art in model reduction, discusses outstanding challenges, and presents possible solution strategies for addressing these challenges.

Model reduction seeks to replace a large-scale system of differential or difference equations by a system of substantially lower dimensions that has nearly the same response characteristics. Two main themes can be identified among several methodologies: (a) balancing based methods, and (b) moment matching methods. Balanced approximation methods are built upon a family of ideas with close connection to the singular value decomposition. These methods preserve stability and allow for global error bounds. Available algorithms for these methods, however, are not suitable for large-scale problems since they have been developed mainly for dense matrix computations. Moment matching methods are based primarily on Padé-like approximations, which, for large-scale problems, have led naturally to the use of Krylov and rational Krylov subspace projection methods. While these moment matching schemes enjoy greater efficiency for large-scale problems, maintaining stability in the reduced order model cannot be guaranteed. Consequently, their use can be problematic at times. Moreover, no a priori global error bounds exist for moment matching schemes.

A current research trend aims at combining these two approaches by deriving iterative methods that incorporate the desirable properties of both of these classes. This paper addresses several important unresolved issues in model reduction of large-scale Linear Time-Invariant (LTI) systems, and extending them to a new class of problems that require adaptive models. It also addresses largescale structured problems that are either time-varying, or which require iterative updating of the initial reduced models to obtain better approximation properties.

\section{Motivating Data Driven Control Applications}

The problems addressed in this paper focus on reduced models for dynamic data-driven systems, which are characterized by model adaptivity and need for maintaining or exploiting a specific type of structure. This can be either due to time variations in the model itself, or due to the fact that the model to be approximated varies at each iterative step of some design loop. 
Large-scale mechanical systems: Consider simulating a structure that is discretely modeled by a large scale finite element formulation such as a longspan bridge, a tall building, or even a car windshield which is subject to various external forces. In tall buildings, for example, these forces could be the result of strong ground motion or wind. Typically, the model is given by second-order systems of equations of the form:

$$
\mathbf{M}(\omega, t) \ddot{\mathbf{x}}(t)+\mathbf{D}(\omega, t) \dot{\mathbf{x}}(t)+\mathbf{K}(\omega, t) \mathbf{x}(t)=\mathbf{f}(t)+\mathbf{B u}(t), \quad \mathbf{y}(t)=\mathbf{C} \mathbf{x}(t) .
$$

Here, $\mathbf{M}(\omega, t)$ is the mass matrix, $\mathbf{D}(\omega, t)$ is the damping matrix, and $\mathbf{K}(\omega, t)$ is the stiffness matrix of the system. All three matrices are assumed to be frequency-dependent and possibly time-dependent. For large-scale structures, the state vector $\mathbf{x}(t)$ is of order $N$, which can reach in the tens of millions. The forcing function $\mathbf{f}(t)$ represents wind and earthquake effects and is of the same dimension. We assume that the structure under consideration is densely instrumented by networked sensing and actuator elements. Further, assuming that a sensor-actuator complex (SAC) is monolithically integrated with a strut system containing controllable dampers that can change the stiffness characteristics of the structure in milliseconds, the control function $\mathbf{u}(t)$, an $m$-dimensional vector, represents the action that can be affected through the smart strut system. Finally, the $p$-dimensional vector $\mathbf{y}(t)$ represents the signals collected via the sensor network. The dimension $N$ of the state vector $\mathbf{x}(t)$, is much larger than $m$ and $p$. The objective of the model reduction problem is to produce a model of the structure that possesses the "essential" properties of the full-order model. Such a reduced order model can then be used for the design of a reduced-order controller to affect real-time control. The reduced-order model may be used to predict the onset of failure, and the reduced-order controller can be used for real-time control of the structure so as to mitigate failure.

There are several preliminary problems that must be solved before we realize an appropriate reduced order model. These include: (i) computing the optimal location of the sensors and actuators, where we could start from a nominal model with $\mathbf{M}, \mathbf{D}$, and $\mathbf{K}$ constant, (ii) improve the dynamical model once the sensor/actuator placements are determined, using validated simulations (via physical laboratory models of the structure) or via measurements performed on the actual structure, if possible, (iii) design a closed-loop controller which is derived from the reduced-order model and, finally, (iv) adapt the reduced order model to the closed-loop configuration of the system (this will change its dynamics and therefore, also its reduced order model). In each of these steps it is useful to have simplified (i.e. reduced-order) models on which the most time consuming tasks can be performed in reasonable time.

\section{Balanced Approximation Methods}

Projection methods have provided effective model reduction algorithms for constructing reduced-order models in the large scale setting. Given a continuoustime state space system:

$$
\dot{\mathbf{x}}(t)=\mathbf{A} \mathbf{x}(t)+\mathbf{B u}(t), \quad \mathbf{y}(t)=\mathbf{C x}(t),
$$


with input $\mathbf{u}(t) \in \Re^{m}$, state $\mathbf{x}(t) \in \Re^{N}$ and output $\mathbf{y}(t) \in \Re^{p}$, one defines a projected model of reduced order $n \ll N$ for a reduced state $\hat{\mathbf{x}}(t) \in \Re^{n}$ :

$$
\dot{\hat{\mathbf{x}}}(t)=\hat{\mathbf{A}} \hat{\mathbf{x}}(t)+\hat{\mathbf{B}} \mathbf{u}(t), \quad \hat{\mathbf{y}}(t)=\hat{\mathbf{C}} \hat{\mathbf{x}}(t),
$$

where

$$
\mathbf{I}_{n}=\mathbf{W}^{T} \mathbf{V}, \hat{\mathbf{A}}=\mathbf{W}^{T} \mathbf{A V}, \hat{\mathbf{B}}=\mathbf{W}^{T} \mathbf{B}, \hat{\mathbf{C}}=\mathbf{C V}
$$

The widely used balanced truncation technique constructs the "dominant" spaces $\mathbf{W}$ and $\mathbf{V}$ such that

$$
\mathcal{P} \mathbf{W}=\mathbf{V} \Sigma_{+}, \quad \mathcal{Q} \mathbf{V}=\mathbf{W} \Sigma_{+}, \quad \mathbf{W}^{T} \mathbf{V}=\mathbf{I}_{n}
$$

where $\Sigma_{+}$is symmetric, positive definite and usually diagonal, and $\mathcal{P}, \mathcal{Q}$ are the controllability and observability gramians, respectively. It follows that $\mathcal{P} \mathcal{Q V}=$ $\mathbf{V} \Sigma_{+}^{2}$ and $\mathcal{Q P} \mathbf{W}=\mathbf{W} \Sigma_{+}^{2}$ and hence $\Sigma_{+}^{2}$ should contain the largest eigenvalues of $\mathcal{P Q}$ or $\mathcal{Q P}$. The gramians are non-singular if and only if the system $\{\mathbf{A}, \mathbf{B}, \mathbf{C}\}$ is controllable and observable. However, the eigenspaces of (5) essentially yields a projected system that keeps those states that are the most observable and controllable simultaneously. This is related to the fact that for a given state $\hat{\mathbf{x}}$, $\hat{\mathbf{x}}^{T} \mathcal{P}^{-1} \hat{\mathbf{x}}$ can be viewed as the input energy needed to steer the system from the zero state to $\hat{\mathbf{x}}$, and $\hat{\mathbf{x}}^{T} \mathcal{Q} \hat{\mathbf{x}}$ can be viewed as the energy of the output resulting from the initial state $\hat{\mathbf{x}}$ (see [5] for a more rigorous discussion).

Efficient algorithms for the large-scale continuous time case have been derived by exploiting the fact that the gramians can be obtained from the Lyapunov equations

$$
\mathbf{A} \mathcal{P}+\mathcal{P} \mathbf{A}^{T}+\mathbf{B B}^{T}=\mathbf{0}, \quad \mathbf{A}^{T} \mathcal{Q}+\mathcal{Q} \mathbf{A}+\mathbf{C}^{T} \mathbf{C}=\mathbf{0} .
$$

and in the discrete-time case from the solution of the Stein equations :

$$
\mathbf{A} \mathcal{P} \mathbf{A}^{T}-\mathcal{P}+\mathbf{B B}^{T}=\mathbf{0}, \quad \mathbf{A}^{T} \mathcal{Q} \mathbf{A}-\mathcal{Q}+\mathbf{C}^{T} \mathbf{C}=\mathbf{0} .
$$

For example, in 46] an iteration called AISIAD was developed. The algorithm alternates between two coupled Sylvester equations that are approximations to the projected equations

$$
\begin{aligned}
\mathbf{A}(\mathcal{P} \mathbf{W})+(\mathcal{P} \mathbf{W}) \mathbf{H}^{T}+\mathbf{B}\left(\mathbf{B}^{T} \mathbf{W}\right) & =\mathcal{P} \mathbf{F}_{w}, \\
\mathbf{A}^{T}(\mathcal{Q} \mathbf{V})+(\mathcal{Q} \mathbf{V}) \mathbf{H}+\mathbf{C}^{T}(\mathbf{C V}) & =\mathcal{Q} \mathbf{F}_{v},
\end{aligned}
$$

where $\mathbf{F}_{w}=\left(\mathbf{I}-\mathbf{W} \mathbf{V}^{T}\right) \mathbf{A}^{T} \mathbf{W}$ and $\mathbf{F}_{v}=\left(\mathbf{I}-\mathbf{V} \mathbf{W}^{T}\right) \mathbf{A V}$. Given a current guess $\mathbf{W}, \mathbf{V}$, the algorithm proceeds by determining $\mathbf{Z}$ by solving $\mathbf{A} \mathbf{Z}+\mathbf{Z H}^{T}+$ $\mathbf{B}\left(\mathbf{B}^{T} \mathbf{W}\right)=\mathbf{0}$, where $\mathbf{H}=\mathbf{W}^{T} \mathbf{A V}$ and $\mathbf{V} \leftarrow \mathbf{V}_{+}$is obtained from $\mathbf{Z}$. $\mathbf{Y}$ is then determined by solving $\mathbf{A}^{T} \mathbf{Y}+\mathbf{Y} \mathbf{H}_{+}+\mathbf{C}^{T}(\mathbf{C V})=\mathbf{0}$ where $\mathbf{H}_{+}=\mathbf{W}^{T} \mathbf{A V}+$ and $\mathbf{W} \leftarrow \mathbf{W}_{+}$is obtained from $\mathbf{Y}$ to maintain relations (5).

The AISIAD iteration is effective, but no convergence results are known. These results may be potentially derived from the fact that one can show (see 
[23]) that $\mathbf{V}$ and $\mathbf{W}$ will span the dominant invariant subspace of the positive definite generalized eigenvalue problems $\left(\lambda \mathcal{P}^{-1}-\mathcal{Q}\right)$ and $\left(\lambda \mathcal{Q}^{-1}-\mathcal{P}\right)$ if and only if they satisfy the respective "Tracemin" conditions:

$$
\begin{aligned}
& \min \text { trace } \mathbf{V}^{T} \mathcal{P}^{-1} \mathbf{V}, \quad \text { s.t. } \mathbf{V}^{T} \mathcal{Q} \mathbf{V}=\mathbf{I}_{n} \text {, } \\
& \min \text { trace } \mathbf{W}^{T} \mathcal{Q}^{-1} \mathbf{W} \text {, s.t. } \mathbf{W}^{T} \mathcal{P} \mathbf{W}=\mathbf{I}_{n} \text {. }
\end{aligned}
$$

\section{Model Reduction for Second Order Systems}

Models of mechanical systems are often of the type (1), where $\mathbf{M}=\mathbf{M}^{T}, \mathbf{D}=\mathbf{D}^{T}$ and $\mathbf{K}=\mathbf{K}^{T}$ are respectively the mass, damping and stiffness matrices, since this represents the equation of motion of the system. In the Laplace domain, the characteristic polynomial matrix $\mathbf{P}(s):=\mathbf{M} s^{2}+\mathbf{D} s+\mathbf{K}$ and the transfer function $\mathbf{G}(s)=\mathbf{C P}^{-1}(s) \mathbf{B}$ appear and the zeros of $\operatorname{det}(\mathbf{P}(s))$ are also known as the characteristic frequencies or poles of the system. Using an extended state $\xi=$ $\left[\mathbf{x}^{T} \dot{\mathbf{x}}^{T}\right]^{T}$, this can be linearized to a generalized state-space model $\{\mathcal{E}, \mathcal{A}, \mathcal{B}, \mathcal{C}\}$ :

$$
\underbrace{\left[\begin{array}{cc}
\mathbf{D} & \mathbf{M} \\
\mathbf{M} & \mathbf{0}
\end{array}\right]}_{\mathcal{E}} \dot{\xi}=\underbrace{\left[\begin{array}{cc}
-\mathbf{K} & \mathbf{0} \\
\mathbf{0} & \mathbf{M}
\end{array}\right]}_{\mathcal{A}} \xi+\underbrace{\left[\begin{array}{c}
\mathbf{B} \\
\mathbf{0}
\end{array}\right]}_{\mathcal{B}} \mathbf{u}, \quad \mathbf{y}=\underbrace{\left[\begin{array}{ll}
\mathbf{C} & \mathbf{0}
\end{array}\right]}_{\mathcal{C}} \xi .
$$

Since $\mathbf{M}$ is invertible, we can also transform this model to the standard statespace form $\left\{\mathcal{E}^{-1} \mathcal{A}, \mathcal{E}^{-1} \mathcal{B}, \mathcal{C}\right\}$, where $\mathcal{E}^{-1} \mathcal{A}=\left[\begin{array}{cc}\mathbf{0} & \mathbf{I} \\ -\mathbf{M}^{-1} \mathbf{K}-\mathbf{M}^{-1} \mathbf{D}\end{array}\right]$ and $\mathcal{E}^{-1} \mathcal{B}=$ $\left[\begin{array}{c}\mathbf{0} \\ \mathbf{M}^{-1} \mathbf{B}\end{array}\right]$. The controllability gramian $\mathcal{P}$ and observability gramian $\mathcal{Q}$ of the state-space model $\left\{\mathcal{E}^{-1} \mathcal{A}, \mathcal{E}^{-1} \mathcal{B}, \mathcal{C}\right\}$ can be computed via the solution of the Lyapunov equations :

$$
\mathcal{E}^{-1} \mathcal{A} \mathcal{P}+\mathcal{P} \mathcal{A}^{T} \mathcal{E}^{-T}+\mathcal{E}^{-1} \mathcal{B B}^{T} \mathcal{E}^{-T}=\mathbf{0}, \quad \mathcal{Q} \mathcal{E}^{-1} \mathcal{A}+\mathcal{A}^{T} \mathcal{E}^{-T} \mathcal{Q}+\mathcal{C}^{T} \mathcal{C}=\mathbf{0}
$$

Moreover, if $\mathbf{C}=\mathbf{B}^{T}$, the transfer function and model (6) are symmetric and the gramians of the state-space model are defined in terms of the solution $\mathcal{G}$ of a single generalized Lyapunov equation :

$$
\mathcal{A G} \mathcal{E}+\mathcal{E} \mathcal{G} \mathcal{A}+\mathcal{B B}^{T}=\mathbf{0}, \quad \mathcal{P}=\mathcal{G}, \quad \mathcal{Q}=\mathcal{E} \mathcal{G} \mathcal{E}
$$

Most model reduction methods use a projection to build the reduced-order model: given a generalized state-space model $\{\mathcal{E}, \mathcal{A}, \mathcal{B}, \mathcal{C}\}$, the reduced-order model is given by $\left\{Z^{T} \mathcal{E} V, \mathbf{Z}^{T} \mathcal{A} \mathbf{V}, \mathbf{Z}^{T} \mathcal{B}, \mathcal{C} \mathbf{V}\right\}$, where $\mathbf{Z}$ and $\mathbf{V}$ are matrices of dimension $2 N \times k$, with $k$ the order of the reduced system. The widely used balanced truncation technique constructs $\mathbf{V}$ and $\mathbf{Z}:=\mathcal{E}^{-1} \mathbf{W}$ such that (5) is satisfied with $\Sigma_{+}^{2}$ the $k \times k$ matrix containing the largest eigenvalues of $\mathcal{P} \mathcal{Q}$.

This technique cannot be applied directly to a second order system since, in general, the resulting reduced order system is no longer a second order system. 
There is a need for adaptive techniques to find reduced order models of second order type. The idea is to choose $k=2 n$ and to restrict the projection matrices $\mathbf{V}$ and $\mathbf{W}$ to have a block diagonal form, where each block is $N \times n$ :

$$
\mathbf{W}=\left[\begin{array}{cc}
\mathbf{W}_{11} & \mathbf{0} \\
\mathbf{0} & \mathbf{W}_{22}
\end{array}\right], \quad \mathbf{V}=\left[\begin{array}{cc}
\mathbf{V}_{11} & \mathbf{0} \\
\mathbf{0} & \mathbf{V}_{22}
\end{array}\right]
$$

which turns out to be a sufficient condition for obtaining an appropriate reduced order model. Notice that for the symmetric case we have automatically $\mathbf{V}=\mathbf{W}$. If we want to impose a block diagonal form, we can, for example, relax the equality conditions in (6).

In this context, the following technical challenges must be addressed: (i) use a relaxation that can be dealt with using Tracemin like optimization methods, such as:

$$
\begin{gathered}
\min \text { trace } \mathbf{V}_{11}^{T}\left[\mathcal{P}^{-1}\right]_{11} \mathbf{V}_{11}+\mathbf{V}_{22}^{T}\left[\mathcal{P}^{-1}\right]_{22} \mathbf{V}_{22} \\
\text { s.t. } \mathbf{V}_{11}^{T}[\mathcal{Q}]_{11} \mathbf{V}_{11}=\mathbf{I}_{n}, \mathbf{V}_{22}^{T}[\mathcal{Q}]_{22} \mathbf{V}_{22}=\mathbf{I}_{n}
\end{gathered}
$$

(ii) define alternative gramians of dimension $N \times N$ for second order systems and analyze their use for model reduction, and (iii) analyze modal approximation based on the polynomial matrix $\mathbf{P}(s)$. One then computes the right and left eigenvectors $\mathbf{P}\left(\lambda_{i}\right) \mathbf{x}_{i}=\mathbf{0}$ and $\mathbf{y}_{i}^{T} \mathbf{P}\left(\lambda_{i}\right)=\mathbf{0}$ via the linearized eigenvalue problem :

$$
\begin{gathered}
{\left[\begin{array}{cc}
\lambda_{i} \mathbf{D}+\mathbf{K} & \lambda_{i} \mathbf{M} \\
\lambda_{i} \mathbf{M} & -\mathbf{M}
\end{array}\right]\left[\begin{array}{c}
\mathbf{x}_{i} \\
\lambda_{i} \mathbf{x}_{i}
\end{array}\right]=\mathbf{0}} \\
{\left[\begin{array}{ll}
\mathbf{y}_{i}^{T} & \lambda_{i} \mathbf{y}_{i}^{T}
\end{array}\right]\left[\begin{array}{cc}
\lambda_{i} \mathbf{D}+\mathbf{K} & \lambda_{i} \mathbf{M} \\
\lambda_{i} \mathbf{M} & -\mathbf{M}
\end{array}\right]=\mathbf{0}}
\end{gathered}
$$

and selects $n$ of them to form the projection matrices $\mathbf{V}_{11}$ and $\mathbf{W}_{11}$.

\section{Time Variance and Adaptivity}

Time variance and adaptivity can be either due to actual time variation of the parameters of the system, or due to an external iteration used to adaptively tune the reduced order system. We first explain how to cope with general time-varying systems and show how these methods can also be used for time invariant or for periodic systems. We then discuss systems that are iteratively adapted within an outer tuning loop.

\subsection{Iterative Schemes}

Reduced order stabilization of a large plant $\mathbf{G}(s)$ amounts to constructing a small order controller $\hat{\mathbf{K}}(s)$ such that the closed loop transfer function $\phi(\mathbf{G}, \hat{\mathbf{K}})=$ 
$\mathbf{G}(s)(\mathbf{I}+\hat{\mathbf{K}}(s) \mathbf{G}(s))^{-1}$ is stable and such that at the same time some performance criterion is optimized.

Most often a crude low-order (PI) controller is available initially. In order to come up with a simple controller which minimizes an appropriate norm of the transfer function between the inputs and the tracking/focusing errors respectively, an iterative procedure is used. The point of the iteration is to successively reduce the order of the high order system until the corresponding optimal controller has low enough order as well as the specified performance properties. Thus, at each reduction step, performance is checked by means of estimating a certain relative error. The rationale of this approach is that the controller should be designed for a small order plant $\hat{\mathbf{G}}_{i}(s)$ using dense matrix techniques. However, this controller will only have a good performance for the original plant $\mathbf{G}(s)$ if the approximation $\hat{\mathbf{G}}_{i}(s)$ is obtained using the closed loop error $\left\|\phi\left(\mathbf{G}, \hat{\mathbf{K}}_{i}\right)-\phi\left(\hat{\mathbf{G}}_{i}, \hat{\mathbf{K}}_{i}\right)\right\|$, where $\phi(\mathbf{G}, \mathbf{K}):=\mathbf{G}(s)(\mathbf{I}+\mathbf{K}(s) \mathbf{G}(s))^{-1}$, hence the need for an outer iteration. An important ingredient for making such an outer iteration tractable is contained in our previous work on the construction of stabilizing controllers using invariant subspace ideas and Riccati iterations for large sparse system models [1].

In this context, one needs to analyze the convergence of the closed loop iteration and find global/local convergence strategies These ideas can be applied to finding reduced order models of plants where certain properties are imposed, such as stability, passivity, or second order model constraints. Finally, this problem can be embedded in a model reduction problem with time-varying coefficients. Therefore it is possible to take advantage of this fact in order to compute the new plant/controller by updating the previous ones.

\section{Simulation and Validation Support}

An important aspect of model reduction and control is to develop a comprehensive environment for validation and refinement. Instrumenting full-scale systems with control mechanisms can be an expensive proposition. For this reason, control techniques must be extensively tested in a simulation environment prior to scale model testing. We have developed an extensive simulation environment for complex structures and demonstrated the power of this environment in the context of several structural impact response simulations (the tragic Pentagon crash on 9/11 is illustrated in Figure 1).

With a view to understanding the nature, extent, and cause of structural damage, we have used our simulation environment to perform high-fidelity crash simulations on powerful parallel computers. A single simulation instance illustrated in Figure 1 with one million nodes (3 d.o.f. per finite element node) over 0.25 seconds of real time takes over 68 hours on a dedicated 8 processor IBM Regatta SMP. A coarser model with $300 \mathrm{~K}$ nodes over $0.2 \mathrm{~s}$ of real time takes approximately 20 hours of simulation time. While we are also working on improving the underlying solvers in our simulation, these computational requirements provide strong motivation for effective error-bounded model reduction. In this 


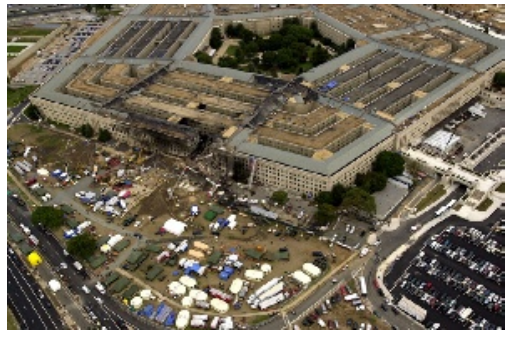

(a) Aerial view of damage to the Pentagon building.

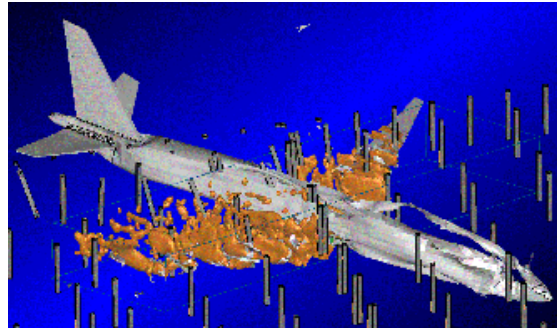

(b) Simulated aircraft impact on $\mathrm{RC}$ columns in the building.

Fig. 1. High fidelity simulation of the Pentagon crash has yielded significant insights into structural properties of reinforced concrete and design of resilient buildings.

context, development of effective preconditioners and iterative solvers, meshing and mesh adaptation techniques, parallel algorithms, and software development and integration pose overarching technical challenges.

\section{Concluding Remarks}

Model reduction is an extremely important aspect of dynamic data-driven application systems, particularly in resource-constrained simulation and control environments. While considerable advances have been made in model reduction, simulation, and control, significant challenges remain. In conjunction with the research activity in areas of sensor networks, actuation mechanisms, and ad-hoc wireless networks, techniques for model reduction and control hold the key to fundamental advances in future generation systems.

\section{References}

1. X. Rao, K. Gallivan, and P. Van Dooren. Efficient stabilization of large scale dynamical systems. In Proceedings IEEE Conference on Computer Aided Control Systems Design, 2000.

2. A. Sameh and Z. Tong. The trace minimization method for the symmetric generalized eigenvalue problem. Journal of Computational and Applied Mathematics, 2000.

3. A. H. Sameh and J. A. Wisniewski. A trace minimization algorithm for the generalized eigenvalue problem. SIAM Journal of Numerical Analysis, 1982.

4. Y. K. Zhou. Numerical Methods for Large Scale Matrix Equations with Applications in LTI System Model Reduction. PhD thesis, Department of Computational and Applied Mathematics, Rice University, 2002.

5. Y. K. Zhou, J. C. Doyle, and K. Glover. Robust and Optimal Control. Prentice Hall, 1995.

6. Y. K. Zhou and D. C. Sorensen. Approximate implicit subspace iteration with alternating directions for lti system model reduction. Technical report, Rice University, 2002. 